\title{
Erratum
}

\section{Accuracy of geometries: influence of basis set, exchange-correlation potential, inclusion of core electrons, and relativistic corrections}

\author{
M. Swart ${ }^{1,2}$, J.G. Snijders ${ }^{1}$ \\ ${ }^{1}$ Theoretische Chemie (MSC), Rijksuniversiteit Groningen, Nijenborgh 4, 9747 AG Groningen, The Netherlands \\ ${ }^{2}$ Current address: Organische en Anorganische Chemie, Vrije Universiteit Amsterdam, De Boelelaan 1083, 1081 HV Amsterdam, \\ The Netherlands \\ Published online: 30 December 2003 \\ (C) Springer-Verlag 2003
}

Theor Chem Acc (2003) 110: 34-41

Due to an unfortunate misunderstanding, the BLAP3 values reported in the original paper were not computed correctly; LAP3 correlation was not included in these calculations, so in fact the values reported as BLAP3 correspond to Becke exchange-only.

The online version of the original article can be found at http://dx. doi.org/10.1007/s00214-003-0443-5 